\title{
New MAC Design to Accommodate Joint Detection Techniques in a MIMO-OFDM-based HIPERMAN System
}

\author{
Linghang Fan, Reza Hoshyar, Sadegh Fazel, Roshano Roberts, Christos Politis, Rahim Tafazolli \\ Mobile Communications Group, Centre for Communication Systems Research, \\ University of Surrey, Guildford, GU2 7XH Surrey, UK \\ \{1.fan, r.hoshyar, m.fazel, r.s.Roberts, c.politis, r.tafazolli\} @eim.surrey.ac.uk
}

\begin{abstract}
One of the key research issues in wireless systems is how to improve the system capacity. MIMO has been proven as an effective method to achieve this. Previously, the focus of MIMO-OFDM research in High Performance Metropolitan Area Network (HIPERMAN) systems was on Space Time Coding (STC) and beamforming. Recently, Multi-User Detection (MUD) has emerged as a novel approach in MIMO-OFDM-based HIPERMAN systems. In this paper, we have proposed a new MAC design, which includes the new and flexible MAC frame structure and an efficient dynamic resource allocation algorithm, in order to accommodate the MUD techniques in uplink transmission in HIPERMAN systems. The performance of the new MAC design has been evaluated via simulation means. The simulation results show that the new MAC design based on MUD can significantly increase the system capacity.
\end{abstract}

\section{Introduction}

A HIPERMAN system, which uses IEEE 802.16 as a prototype, is a Broadband Fixed Wireless Access (BFWA) system [1]. It has advantages of rapid deployment, large range capabilities and low maintenance cost. However, like all the other wireless systems, HIPERMAN has limited capacity per base station. Therefore, how to improve the system capacity is a key research issue in HIPERMAN systems [2].

Since the MIMO techniques allow more users to be served at the same time in the same frequency, they are emerging as a novel approach for spectrally efficient terrestrial HIPERMAN systems. In the recent years, the focus of MIMO-OFDM research in HIPERMAN was on STC and beamforming [3], and some of them have been standardised [4].

Since MUD allows the parallel operation of multiple radio links and has very good performance in NLOS environment, using MUD in OFDM-based HIPERMAN system to improve the system capacity is emerging as a new research area. In this paper, we have investigated the effect of MUD on the HIPERMAN MAC design, and proposed a new HIPERMAN MAC design to accommodate MUD techniques in uplink transmission. Based on a flexible MAC frame structure, a dynamic resource allocation algorithm is proposed to intelligently construct the MAC frame and therefore maximise the system capacity.

In this paper, we firstly describe the proposed HIPERMAN MAC frame structure. Then, a novel dynamic resource allocation algorithm is proposed to accommodate the MUD techniques. Following this, we evaluate the performance of the proposed MAC design via simulation. Finally, conclusions are drawn.

\section{New MAC Design}

By introducing MUD techniques into the HIPERMAN systems, it will have significant impacts on MAC design, especially on MAC frame structure, scheduling and resource allocation algorithm and the interface between the MAC and physical layers.

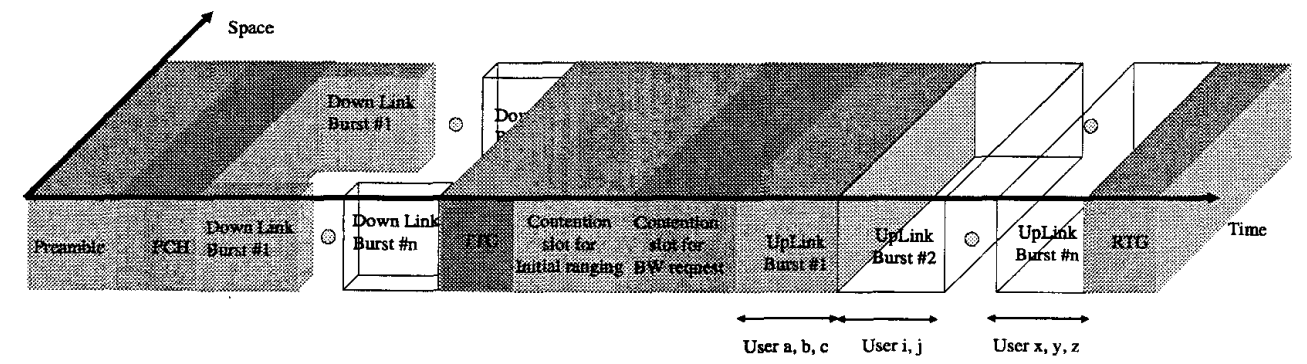

Figure 1 MAC Frame Structure Using MIMO techniques 
Figure 1 shows the proposed MAC frame structure. Like the current design, the MAC frame is divided into uplink and downlink sub-frames. Frame Control Header (FCH) is used to specify the whole MAC frame. It contains information on how to access the uplink and downlink, and modulation and coding schemes of the allocated data. In this paper, the proposed changes on $\mathrm{FCH}$ are specified and new Information Elements (IEs) are introduced. The proposed MAC frame not only can accommodate the MUD techniques, but also is compatible with the current design. One of the major advantages of our proposal is that it does not result in major modifications to current standards.

By adopting MUD techniques in HIPERMAN, each user will have a unique spatial channel signature, this signature is a complex vector, and depends on the propagation environment and multi-path fading channel realization. The performance of exploited MUD technique will strongly depend on the amount of correlation between users' spatial signature. In general, higher User Signature Correlation (USC) values are detrimental, especially when there are a high number of simultaneous users being served. The system capacity depends on the users' transmit power, users' signal-to-interference-plus-noise ratio (SINR), the number of receiver antennas at base station and the correlations between the users. The SINR of each user can be appropriately used on selection of appropriate combination of users for joint transmission. The SINR on a single user can be expressed as follows:

$$
\begin{aligned}
\operatorname{SINR}(i) & =\frac{P_{i}}{I_{i}+N} \\
& =\frac{P_{i}}{\left.\sum_{i \neq j}\left(\frac{1}{N_{s c}} * \sum_{k=1}^{N_{s c}-1}\left(\rho_{i j}(k)\right)^{2} * P_{j}\right)\right)+N}
\end{aligned}
$$

SINR(i) is the SINR of the User $i, P_{i}$ and $P_{j}$ are the received powers of Users $i$ and $j$, respectively. $\rho_{i j}$ is the cross correlation between Users $i$ and $j$ spatial signatures, $I_{i}$ is the interference from other users on user $i, N$ is the additive noise in front end of to the User I receiver, and finally $\mathrm{N}_{\mathrm{sc}}$ is the number of sub-carriers. As shown in the above formula, SINR(i) depends on $\rho_{\mathrm{ij}}$. It means that the users' SINR are highly related to their spatial separation. The higher the users' spatial correlation, the lower their SINR will be.

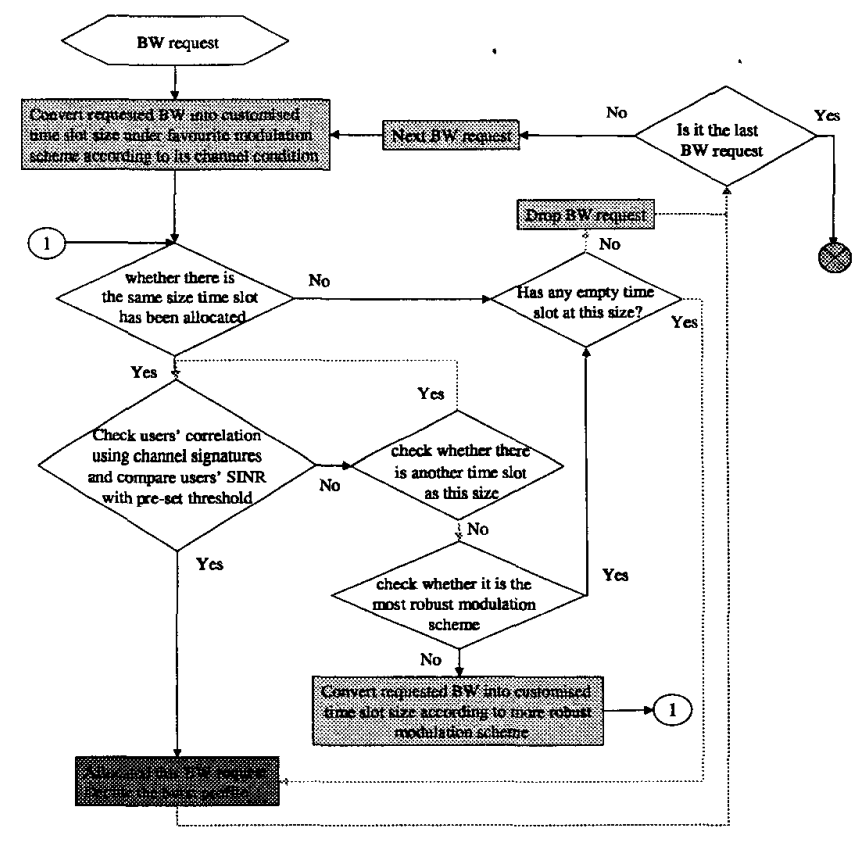

Figure 2 Dynamic Resource Allocation Algorithm 
Since the users' spatial characteristics are time varying, an efficient dynamic resource allocation algorithm is required to accommodate MUD and thus increase the system capacity. The scheduler in base station, which implement the dynamic resource allocation algorithm, will monitor the SINR between all possible pairs of users and decide which users are to be served simultaneously based on their SINR and the cross correlation of channel signatures between the users.

The dynamic resource allocation algorithm is specified as shown in Figure 2. It main principle is as follows:

Based on the channel signature matrix, each user's SINR can be obtained. When a new bandwidth request is under processing, the scheduler will find a time burst and checks whether the new user's channel signature is consistent in terms of the adopted scheduling measure (i.e. SINR of all users of the corresponding channel signature matrix) with the channel signatures of already allocated users in this time burst. This means that the scheduler will re-calculate the adopted measure related to the new set of already allocated user and new tentatively added user, and then compare with a pre-set threshold. If the re-calculated measure is acceptable (i.e. all users' SINR to be above the corresponding threshold), the new user's requested bandwidth will be allocated and the burst profile will be updated. Otherwise, the scheduler will check whether the new user's bandwidth request can be allocated in another burst or not.

The proposed dynamic resource allocation algorithm has the following advantages:

- When it receives a bandwidth request, it goes through all the relevant bursts to see with which group of users the new user can be simultaneously transmitted and do not degrade their performance. This will maximise the opportunity to allocate users and significantly increase the system's capacity.

- Since the flexible modulation scheme is used, it improves the chance of new bandwidth requests to be allocated.

Simulation Evaluations

\begin{tabular}{|l|c|}
\hline Frame length & $10 \mathrm{~ms}$ \\
\hline Cyclic prefix & $1 / 32$ \\
\hline $\mathrm{N}_{\mathrm{FFT}}$ & 256 \\
\hline Bandwidth & $7 \mathrm{MHz}$ \\
\hline Duration of one OFDM symbol & $33 \mu \mathrm{s}$ \\
\hline Modulation schemes & QPSK, 16-QAM, 64-QAM \\
\hline
\end{tabular}

Table 1 - Main Parameters used in System Level Simulation

The proposed MAC layer, including the new MAC frame structure and dynamic resource allocation algorithms, has been simulated and evaluated via simulation. A system level simulator and a link level simulator, which is used to perform physical layer simulation, are used during the simulation, and the results from the link level simulator are used as the input to the system level simulator. The parameters used in the system and link level simulation are listed in Table 1 and 2.

\begin{tabular}{|l|l|}
\hline Number of OFDM sub-carriers & 256 \\
\hline Number of Data Sub-carrier & 200 \\
\hline Coding and Modulation technique & Uncoded QPSK \\
\hline Power of users & Equal power \\
\hline Channel & $2 \times 2$ MIMO 6-tap SUI-5 Rayleigh Fading \\
\hline Correlation & $\begin{array}{l}\text { Without Correlation: Case 0 } \\
\text { With Correlation: Case 1, 2, 3, and 4 }\end{array}$ \\
\hline
\end{tabular}

Table 2 HIPERMAN link layer chain setting used for performance evaluation of MUD

To study the impacts of using MUD techniques in HIPERMAN, we have specifically constructed a cell, whose geography are made of several typical scenarios and a group of users has been located in the cell. In these scenarios, we use different users' positions, different distances between the users, different scatters' separation angles and different scatters' positions. Based on the results obtained from the typical scenarios, we can get a clear picture about the impact of cell geography and users' positions in the cell on the users' spatial correlation and users' SINR values. For example, the results show that, for the uplink multi-user joint detection, Rx correlation is a dominant factor in the user's spatial correlation. Tx correlation is also important but is not a dominant factor. When multi-user joint detection techniques are applied in the uplink, the user's spatial correlation is highly related to the cell's geography and users' positions in the cell. 
Based on the above studies, the results from the link level simulator are used as the input to the system level simulator. Based on the system level simulation, we study the impact of new MAC design on system capacity, and also compare the system capacity under different scenarios, such as changing the uplink sub-frame length.

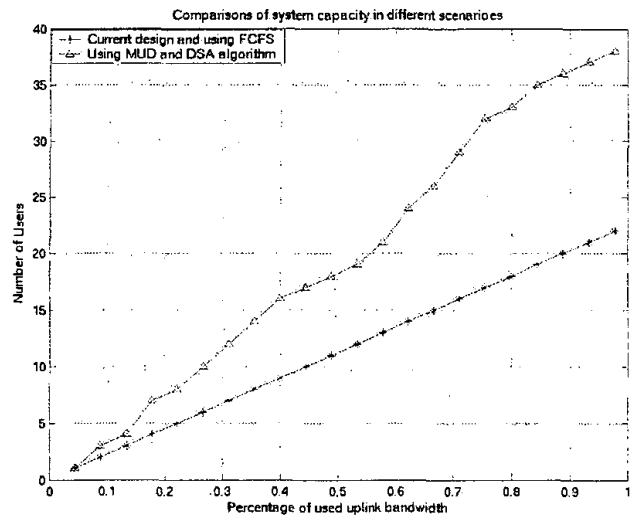

Figure 3 Comparisons of System Capacity between Different MAC Designs

(90 OFDM symbols are allocated to the uplink)

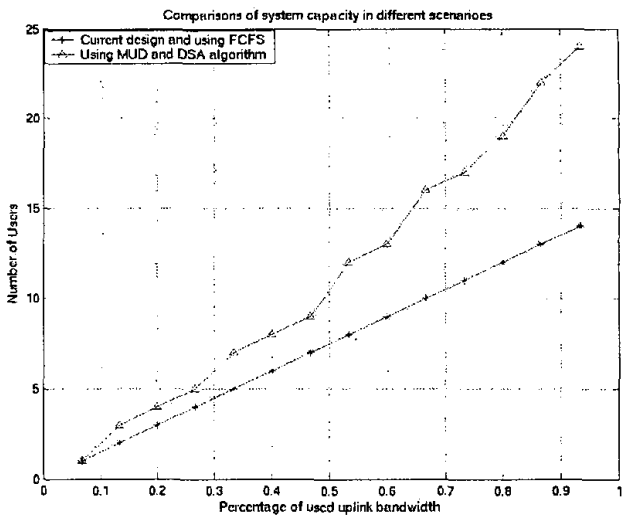

Figure 4 Comparisons of System Capacity between Different MAC Designs

(60 OFDM symbols are allocated to the uplink)

Figure 3 shows the comparison of system capacity between different MAC designs. The "dash-triangle" line represents the system capacity where our proposed new MAC design with dynamic resource allocation is used to exploit MUD. When 90 OFDM symbols are allocated to the uplink and QPSK is used, overall 38 users can be accommodated. The "dash-star" lines represent the system capacity where First-Come-First-Served algorithm is used without multi user transmission. For the same condition, when 90 OFDM symbols are allocated to the uplink and QPSK is used, overall 22 users can be accommodated. This shows that the proposed MAC design with multi user dynamic resource allocation achieves $73 \%$ increase in system capacity.

Since HIPERMAN is a TDD system, the resources allocated between uplink and downlink subframes are not fixed. Figure 4 shows the comparison of system capacity between different MAC designs when 60 OFDM symbols allocated to the uplink subframe. Similar to Figure 3, the "dash-triangle" line represents the system capacity with multi user dynamic resource allocation. When 60 OFDM symbols are allocated to the uplink and QPSK is used, overall 24 users can be accommodated. The "dash-star" line represents the system capacity where First-Come-First-Served algorithm is used without MUD. Under the same conditions overall 14 users can be accommodated. This translates to $72 \%$ increase in system capacity thanks to the proposed MAC design. This confirms the trend in system capacity increase as observed in the 90 OFDM symbol case.

\section{Conclusions}

In this paper, we have proposed a new MAC design, which includes the MAC structure and a dynamic resource allocation algorithm to accommodate the MUD techniques, which is a novel approach in HIPERMAN systems research. The simulation results prove that the new MAC design can effectively take advantage of MUD techniques and therefore significantly increase the system capacity. In the future, we will further develop our design and compare our design with more other proposals.

References

[1] C. Eklund, R. Marks, K.L. Stanwood and S. Wang, "IEEE standard 802.16: A Technical Overview of the WirelessMAN Air Interface for Broadband Wireless Access”, IEEE Communications Magazine, June 2002.

[2] ETSI, "P802.16/D5c-2002, HIPERMAN Draft," Tech. Rep., European Telecommunications Standards Institute, July 2002, Version D5c.

[3] F. Shad, T.D. Todd, V. Kezys and J. Litva: "Dynamic Slot Allocation (DSA) in Indoor SDMA/TDMA Using a Smart Antenna Basestaion”, IEEE Transactions on Networking, Vol.9, No. 1, February 2001.

[4] ETSI, TS 102177 v.0.0.6, Broadband Radio Access Network, HIPERMAN, Part 1: Physical Layer, $2003-02$. 


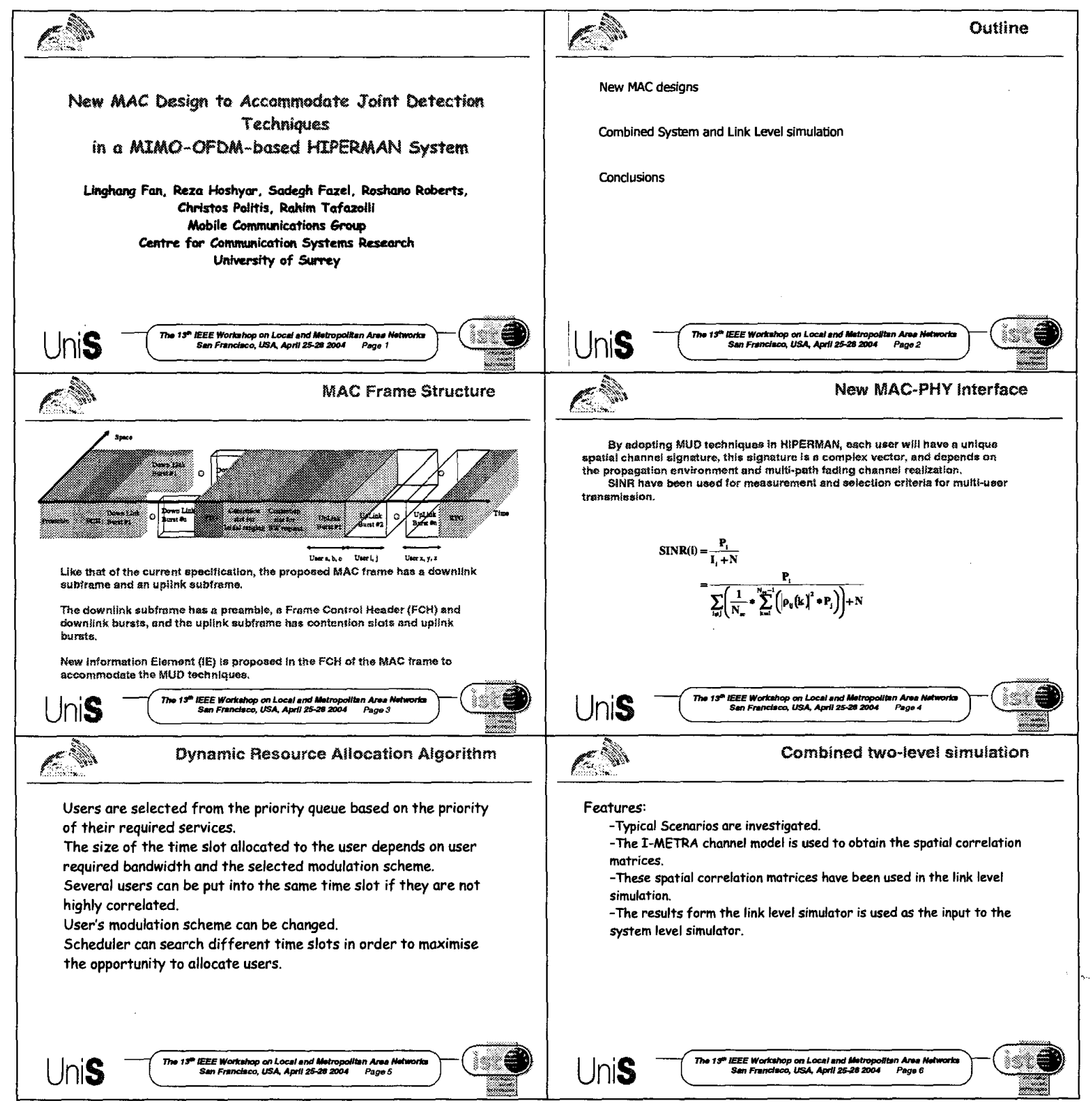




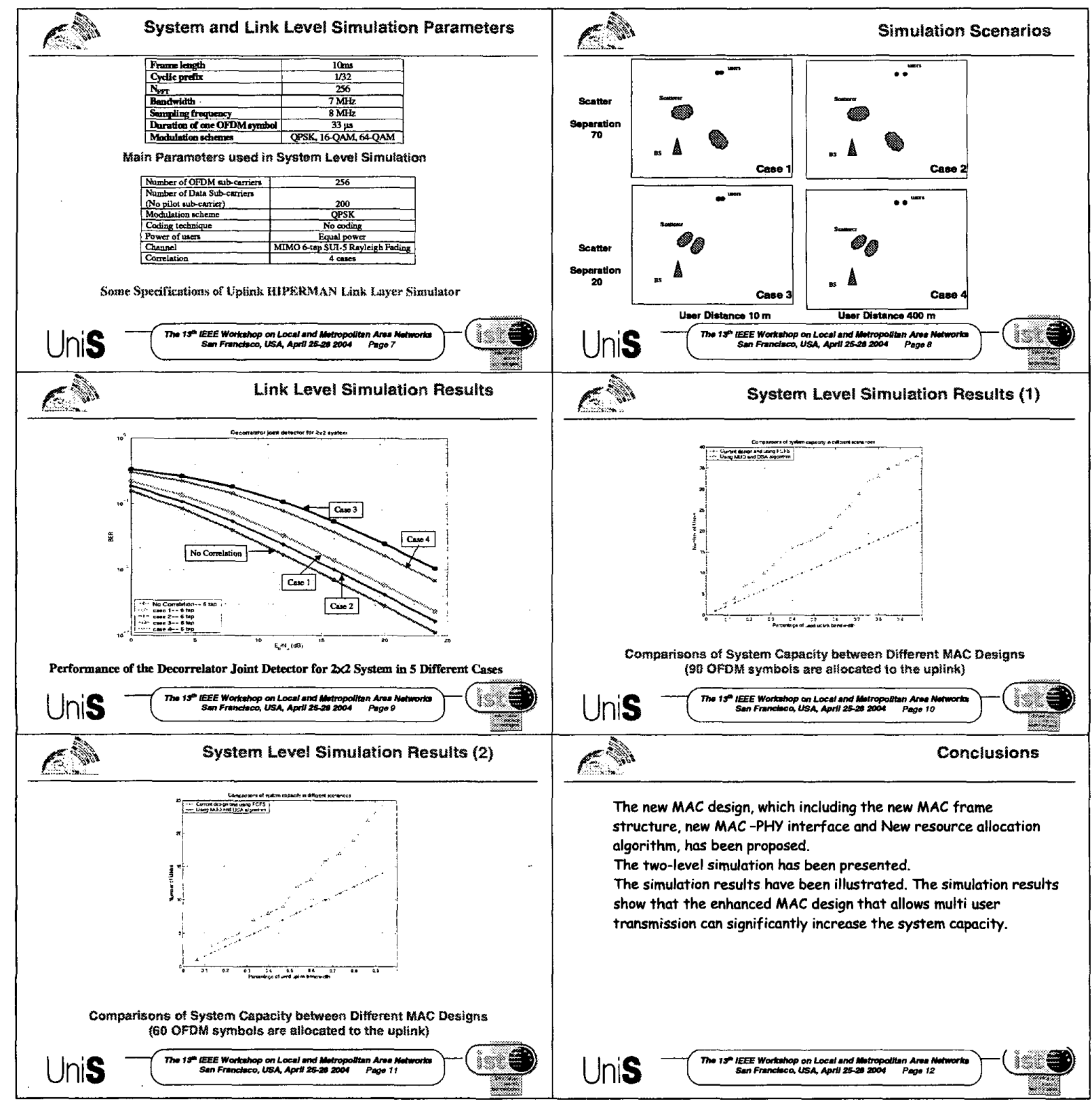

